\title{
Schizophrenia-related cognitive dysfunction in the Cyclin-D2 knockout mouse model of ventral hippocampal hyperactivity
}

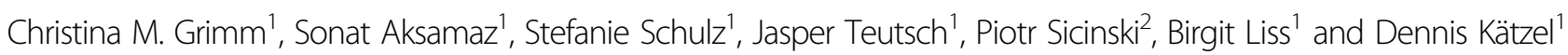

\begin{abstract}
Elevated activity at the output stage of the anterior hippocampus has been described as a physiological endophenotype of schizophrenia, and its development maps onto the transition from the prodromal to the psychotic state. Interventions that halt the spreading glutamatergic over-activity in this region and thereby the development of overt schizophrenia could be promising therapies. However, animal models with high construct validity to support such pre-clinical development are scarce. The Cyclin-D2 knockout (CD2-KO) mouse model shows a hippocampal parvalbumin-interneuron dysfunction, and its pattern of hippocampal over-activity shares similarities with that seen in prodromal patients. Conducting a comprehensive phenotyping of CD2-KO mice, we found that they displayed novelty-induced hyperlocomotion (a rodent correlate of positive symptoms of schizophrenia), that was largely resistant against D1- and D2-dopamine-receptor antagonism, but responsive to the mGluR2/3-agonist LY379268. In the negative symptom domain, CD2-KO mice showed transiently reduced sucrose-preference (anhedonia), but enhanced interaction with novel mice and objects, as well as normal nest building and incentive motivation. Also, unconditioned anxiety, perseveration, and motor-impulsivity were unaltered. However, in the cognitive domain, CD2knockouts showed reduced executive function in assays of rule-shift and rule-reversal learning, and also an impairment in working memory, that was resistant against LY379268-treatment. In contrast, sustained attention and forms of spatial and object-related memory that are mediated by short-term habituation of stimulus-specific attention were intact. Our results suggest that CD2-KO mice are a valuable model in translational research targeted at the pharmacoresistant cognitive symptom domain in causal relation to hippocampal over-activity in the prodrome-topsychosis transition.
\end{abstract}

\section{Introduction}

The re-creation of endophenotypes of psychiatric diseases in animal models has been proposed as a promising strategy to meet the current challenge of identifying rodent models with predictive validity for drug discovery ${ }^{1}$. In schizophrenia, hyperactivity of the output stages of the anterior hippocampus represents a physiological

\footnotetext{
Correspondence: Dennis Kätzel (dennis.kaetzel@uni-ulm.de)

${ }^{1}$ Institute for Applied Physiology, UIm University, Ulm, Germany

2Dana-Farber Cancer Institute and Harvard Medical School, Boston, MA, USA

These authors contributed equally: Sonat Aksamaz, Stefanie Schulz
}

endophenotype that is unique in predicting disease progression from the prodromal state to overt schizophrenia (psychosis) $^{2,3}$. More precisely, baseline metabolic hyperactivity in the anterior CA1 region is elevated in prodromal patients that will develop psychosis in the near future, compared to individuals that do not. The development of hyperactivity in the anterior subiculum, the output stage of the hippocampus that is excited by CA1, in turn, coincides with the onset of psychosis and is accompanied by atrophy of the anterior hippocampus, which might manifest the disease state irreversibly ${ }^{2}$. 
Therefore, interventions that ameliorate anterior hippocampal hyperactivity in the prodromal state and thereby halt further disease progression could be a promising therapeutic strategy ${ }^{4}$.

To support this research, rodent models that display hyperactivity of the ventral hippocampus ( $\mathrm{vHC}$, which is homologous to the human anterior hippocampus) are desired. In rats, rodent correlates of the positive symptom domain were induced by direct electrical or chemical stimulation of the $\mathrm{vHC}^{5-7}$. Alternatively, developmental manipulations such as pre-natal methylazoxymethanol acetate (MAM) injections ${ }^{8,9}$, which lead to $\mathrm{vHC}$ hyperactivity later (but also prefrontal aberrations), have been used. It is well established, that such $\mathrm{vHC}$ hyperactivity entails a hyper-dopaminergic state of the mesolimbic projection $^{5,8}$, and thereby deficits of latent ${ }^{9,10}$ and prepulse inhibition ${ }^{6,7,9}$, and enhanced novelty- and amphetamine-induced hyperlocomotion ${ }^{5,7,8}$.

Other studies focused largely on the contribution of a hypofunction of inhibitory interneurons to $\mathrm{vHC}$ hyperactivity. It had been suggested previously, that a homeostatic downregulation of the GABAergic output from parvalbumin-positive (PV) interneurons of the CA1region of the hippocampus might lead to an effective disinhibition of the pyramidal neurons of CA1, which would then over-activate the excitatory projection neurons of the ventral subiculum, that project to multiple target areas in the brain that are relevant for affective and cognitive functions ${ }^{11}$. Chemogenetic silencing of $\mathrm{vHC}$ interneurons in mice ${ }^{12}$ as well as pharmacological vHC disinhibition with picrotoxin in rats ${ }^{13}$ could indeed provoke some impairments in the positive and the cognitive symptom domains (especially hyperlocomotion and deficits of spatial short-term memory).

A different set of studies focused on the Cyclin-D2 knockout mouse model $\left(C c n d 2^{-/-} \text {, or CD2-KO }\right)^{14}$ that displays a reduced number of PV-cells in the hippocampus (20-40\% reduction), particularly in CA1, and some areas of the neocortex (10-30\%), but not the medial prefrontal cortex ${ }^{15,16}$. While the deletion of Ccnd2 affects cell development in the subventricular zone of the medial ganglionic eminence where PV-cells originate from, somatostatin-positive interneurons that derive from the same region, are not decreased in their numbers in the adult $\mathrm{CD} 2-\mathrm{KO}$ brain ${ }^{16}$. Cerebral blood volume (CBV) measurements in resting-state functional magnetic resonance imaging demonstrated that these animals show hyperactivity of the dentate gyrus, CA2/CA3 and CA1 of the $\mathrm{vHC}^{15}$ - but not the ventral subiculum; a pattern that is more akin to the prodromal state (see above) in which patients already present with cognitive symptoms. However, schizophrenia-related cognitive deficits have not yet been systematically examined in this mouse line (see Table 1 for a summary of previous behavioural assessments of $C c n d 2^{-/-}$mice) nor in most other rodent models of ventral hippocampal hyperactivity (for exceptions see ref. $\left.{ }^{13-17}\right)$. Most relevant to schizophrenia is the finding, that $\mathrm{CD} 2-\mathrm{KO}$ mice are less efficient in their search strategies in the water-maze and in spatial forms of cognitive flexibility, while basic spatial learning is intact ${ }^{18-}$ 21 .

Importantly, CD2-KO mice also show a lack of adult neurogenesis in the dentate gyrus ${ }^{20}$, which adds to its translational value given that indicators of reduced neurogenesis were also found in post-mortem brains from patients with schizophrenia ${ }^{22}$. Furthermore, PV-cell hypofunction could be contributing to the neurogenesis deficit, since the innervation of new-born cells by PVinterneurons is critical for their survival and development ${ }^{23}$. In reverse, new-born granule cells activate strong dentate gyrus-feedback- and CA3-feedforward inhibition $^{24,25}$, implying that reduced neurogenesis alone might lead to hippocampal hyperactivity. Importantly though, physiological and some behavioural aberrations in the $\mathrm{CD} 2-\mathrm{KO}$ mouse, could be rescued by implantation of GABAergic precursor cells specifically into the ventral hippocampus, pin-pointing this part of the hippocampus and its over-activity as the primary cause of those abnormalities $^{15}$. This suggests that the $C c n d 2^{-/-}$mouse model might be well-suited to study the role of ventral hippocampal hyperactivity in the causation of schizophrenia-related deficits. To that end, we have subjected $C c n d 2^{-/-}$mice to a large battery of behavioural tests in the positive, negative, and cognitive symptom domains.

\section{Methods \\ Animals}

Cyclin-D2 knockout mice (Ccnd2 $2^{-/-}$, CD2-KO; 18 males, 12 females) ${ }^{14}$ and wild-type littermate controls (19 males, 9 females) were generated by crossing heterozygous $C c n d 2^{-/+}$mice. Animal numbers used for each test are stated in the respective figure panels, where appropriate, and in Supplementary Tables 1 and 3. The average age of the cohort at the time of each test is given in Supplementary Table 2. Sizes of same-gender samegenotype subgroups were chosen to be around 10 according to common standards in behavioural neuroscience ${ }^{26}$ and, where possible, based on our own biometric estimates using prior data from the same tests ${ }^{27}$. If logistically possible, sub-group sizes of a single gender and genotype were increased to up to 19 to improve statistical power. Animals were group-housed in Type II-Long individually ventilated cages (Greenline, Tecniplast, G), enriched with sawdust, sizzle-nest ${ }^{\mathrm{TM}}$, and cardboard houses (Datesand, UK), and subjected to a $13 \mathrm{~h} \mathrm{light} \mathrm{/} 11 \mathrm{~h}$ dark cycle. All experiments conformed to the German Animal Rights Law (Tierschutzgesetz) 2013 and were 
Table 1 Overview over previous behavioural studies in CD2-KO mice. Numbers refer to citations in the reference list

\begin{tabular}{|c|c|c|}
\hline Psychological Function & Behavioural test & Phenotype \\
\hline \multirow[t]{4}{*}{ Spatial associative learning } & Water-maze & Normal $^{20}$ but less precise/efficient searching ${ }^{18}$ \\
\hline & Plus-maze & Normal ${ }^{19}$ \\
\hline & Barnes maze & Normal, but more errors during training ${ }^{53}$ \\
\hline & Place preference (IntelliCage) & Normal $^{20}$ \\
\hline Spatial reversal learning & Water-maze & Impaired (delayed acquisition/higher perseverance) ${ }^{18}$ \\
\hline Spatial reversal learning & Plus-maze & Normal ${ }^{19}$ \\
\hline Spatial rule-shift learning & Plus-maze (ego $\rightarrow$ allocentric) & Impaired (delayed acquisition, but not due to perseverative errors) ${ }^{19}$ \\
\hline \multirow[t]{3}{*}{ Aversive associative learning } & Cue fear-conditioning & $\begin{array}{l}\text { Normal }{ }^{20,21} \text {, or enhanced freezing in novel context } \mathrm{t}^{21} \text {, or reduced freezing in } \\
\text { novel context after repeated cue presentation }{ }^{15}\end{array}$ \\
\hline & Context fear-conditioning & Normal $^{20,21}$ or reduced ${ }^{15}$ freezing \\
\hline & Trace fear-conditioning & Improved ${ }^{20}$ \\
\hline Operant learning & Nose-poking for reward & Normal $^{20}$ \\
\hline Procedural learning & (various) & Normal $^{20}$ \\
\hline Anhedonia & Sucrose preference & Normal, but transient anhedonia (delayed preference) ${ }^{21}$ \\
\hline Behavioural despair & Forced swim test, Tail suspension test & Mildly anti-depressive (reduced immobility) ${ }^{54}$ \\
\hline Nesting & Nest building & Reduced $^{21}$, potentially due to avoidance of novel nesting material ${ }^{15}$ \\
\hline Sensitivity to psychostimulants & Amphetamine-induced locomotion & Enhanced $^{15}$ \\
\hline Stereotypy & Stereotypic movements & Decreased $^{21}$ \\
\hline \multirow[t]{4}{*}{ Processing of novelty } & Novelty-induced locomotion & Enhanced $^{21}$ or normal ${ }^{20}$ \\
\hline & Novelty-induced exploration & Enhanced $^{21}$ \\
\hline & $\begin{array}{l}\text { Exploration of novel material, incl. } \\
\text { bedding }\end{array}$ & Avoidance of novel material ${ }^{15}$ \\
\hline & Digging and marble burying & Reduced $^{21}$ \\
\hline \multirow[t]{2}{*}{ Unconditioned anxiety } & Elevated Plus-maze & Normal $^{20}$ \\
\hline & Open field & Normal $^{20}$ \\
\hline Olfaction & (Various) & Normal $^{15}$ or reduced $^{20}$ \\
\hline Vision, tactile sense & (Various) & Normal $^{15}$ \\
\hline Posture, gait, muscle tone & (Various) & Normal ${ }^{15}$ \\
\hline
\end{tabular}

approved by the Federal Ethical Review Committee (Regierungsprädisium Tübingen) of Baden-Württemberg.

\section{Behavioural tests}

Detailed protocols for all behavioural tests are given in Supplementary Methods and the majority of protocols were as previously described ${ }^{27}$. Light levels were set to 100 lux for all tasks in open arenas. In brief, in the novel-object recognition test, mice were exposed to two identical objects, A1 and A2, in the sample phase $(10 \mathrm{~min})$ and after a delay of $2 \mathrm{~min}$ - to an identical copy of the familiar object, A3, and a novel object, B1, in the test phase (5 min). Direct physical interaction between the snout or whiskers with the object were scored manually by an observer blind to genotype, and the extent of novel-object recognition was determined according the ratio of interaction with B1 to interaction with B1 and A3 combined. Spatial novelty preference testing in the Y-maze was conducted analogously: animals explored the start arm and a pseudo-randomly selected goal arm in the sample phase ( $5 \mathrm{~min}$ ), while they had access to all arms (including the previously unvisited novel arm) in the test phase (2 min) that followed after a 1 min-delay period during which the sawdust in the maze was mixed to remove olfactory landmarks. In the T-maze test of working memory, 10 trials were conducted per day in fooddeprived (85-90 \% of free-feeding weight) animals. Each trial consisted of a sample run with a forced choice into 
one of the goal arms, and a choice run in which the previously unvisited arm was baited and its visit was counted as a correct choice. Animals were trained up over 5 days in a blocked paradigm with a delay between sample and choice run of $5 \mathrm{~s}$ and an inter-trial interval (ITI) of 3-10 min. Subsequently, the protocol was altered every second day starting with a massed design with a delay of $1 \mathrm{~s}$ and an ITI of $25 \mathrm{~s}$, followed by protocols in a blocked design with delays of 5,20 , and $60 \mathrm{~s}$. The assessment of spatial reference learning, rule-shift and rule-reversal were conducted in a plus-maze with transparent walls where the start arm (North or South) was chosen pseudorandomly and one of the goal arms (East or West) was baited according to the respective rule and its visit counted as a correct choice. 10 trials were given per day with a delay of 5-10 min between them. Animals were first trained up on an egocentric rule (turn left or turn right). After achieving the performance criterion of $85 \%$ correct choices in 20 consecutive trials, animals were switched to an allo-centric rule (go West or go East) to assess spatial rule-shift learning. After reaching the same $85 \%$ performance criterion the allo-centric rule was reversed (e.g. from go West to go East, or vice versa) to assess reversal learning.

Unconditioned anxiety was measured on the elevated plus-maze, according to the preference with which animals explored the two open vs the two closed arms. Novelty-induced locomotion was assessed by letting animals explore a new Typ-III-cage (Tecniplast, G) with clean sawdust for $2 \mathrm{~h}$. Movement was video-tracked using ANY-maze (San Diego Instruments, CA, USA) in the Ymaze, the elevated plus-maze, and during novelty-induced locomotion. Reciprocal social interaction was assessed in similar cages but with darker walls (Tecniplast), into which a younger adult stimulus mouse of the same sex and strain had been introduced. Social interactions (sniffing, taxing with snout or whiskers, but not aggression or sexual behaviour) were scored offline manually and blind to genotype over a period of $16 \mathrm{~min}$ in $2 \mathrm{~min}$ intervals.

Sucrose preference was assessed overnight in animals that were single-housed on the first day of the testing sequence, on which they were presented with two bottles of drinking water. Over the 4 subsequent nights one of the two bottles contained either $1 \% \mathrm{w} / \mathrm{v}$ ( 2 nights) or $10 \% \mathrm{w} /$ $\mathrm{v}$ (2 nights) sucrose in drinking water, while only the water bottle remained in the cages during the day and for the sixth night. During this last night, animals received a Nestlet $^{\mathrm{tm}}$ (Datesand) of 2.3-2.6 g, and nests were scored according to an established nest building rating system ${ }^{28}$, next morning.

For the 5-choice-serial reaction time task (5-CSRTT) mice were trained up in operant chambers (Med Associates Inc., VT, USA) as previously described ${ }^{27}$ over 4 stages with increasing attentional difficulty until mice reached a consistent performance ( $\geq 80 \%$ accuracy, $\leq 50 \%$ omissions, $\geq 30$ trials) on the stage 4 baseline protocol where the stimulus duration (SD) was $4 \mathrm{~s}$ and the inter-trial interval (ITI) time was $5 \mathrm{~s}$. Subsequently, various protocols to challenge either sustained attention (by reduction of the SD to $1 \mathrm{~s}$ or distraction, or both) or impulse control (by extension of the ITI to $9 \mathrm{~s}$ ) were applied over 2 consecutive days, followed by 1-3 d of baseline training before the next challenge. Afterwards, mice were trained on a simplified version of this task where only hole 2 or 4 was illuminated in each trial until mice showed an accuracy of $\geq 80 \%$ in two consecutive sessions. Subsequently, the rule was shifted from a cued to a spatial version, whereby only hole 2 (or, in other mice, hole 4) was rewarded irrespective if hole 2 or 4 was illuminated in a given trial. To assess rule-shift learning in this operant paradigm, mice were trained until they had reached a criterion of $80 \%$ accuracy across trials in which the correct hole was unlit on 3 consecutive days.

Condensed milk diluted with drinking water was used as reward on rewarded maze tasks, strawberry milk was used in operant box paradigms. Experimenters were blind to genotype in all instances of manual scoring (nest building, social interaction, novel-object recognition) and in all maze-based assays.

\section{Behavioural pharmacology}

To assess the effects of the D1R-antagonist SCH23390, the D2R-antagonist raclopride and the mGluR2/3-agonist LY379268 on novelty-induced locomotion in CD2-KO mice, each drug was injected i.p. at an injection volume of $10 \mathrm{ml} / \mathrm{kg}$ before an $1 \mathrm{~h}$ exposure in a novel open-field, identical to those used for the prior assessment of noveltyinduced locomotion (see above). S(-)raclopride- $(+)$ tartrate salt (Sigma, G) was diluted in $0.3 \%$ TWEEN80/ saline and injected $60 \mathrm{~min}$ before the start of the run. SCH23390 hydrochloride and LY379268 disodium salt (Tocris, G) were diluted in saline and injected $30 \mathrm{~min}$ or $45 \mathrm{~min}$, respectively, before the run. For dose-response assessments of these drugs, separate adult male C57BL/6 cohorts were used in a between-subject design (4-8 mice per subgroup); either raclopride, SCH23390 or LY379268 were injected $30 \mathrm{~min}, 30 \mathrm{~min}$, or $45 \mathrm{~min}$, respectively, before a $30 \mathrm{~min}$ exposure to the novel open-field, followed by injection of $0.2 \mathrm{mg} / \mathrm{kg}$ MK-801 maleate (in saline) ${ }^{27}$ and another $60 \mathrm{~min}$ of locomotor monitoring. For the $T$ maze test, LY379268 was injected 50-60 min before testing commenced. Additionally, amphetamine-induced locomotion was tested by injection of $\mathrm{D}$-amphetamine hemisulfate (Sigma; diluted in saline), $30 \mathrm{~min}$ after an acclimatisation period in the novel open-field, immediately followed by another hour of post-injection exposure. Latin-square designs with a fixed decreasing order of 
doses, counterbalanced for starting dose and genotype, were used for all within-subject studies.

\section{Analysis}

Within-subject data was analysed by repeated-measures ANOVA, and between-subject data with univariate ANOVA (subsequently termed "ANOVA" only), one-way ANOVA in cases of one-factorial design with more than 2 groups, and $t$-tests, or - in cases of non-parametric data the Mann-Whitney-U-Test (termed MWU) in cases of individual comparisons between two groups only, as appropriate. All graphs display mean \pm s.e.m. Each experiment was conducted once in the cohorts of the stated sizes ( $\mathrm{n}$ ); for the confirmation of the effects of raclopride and LY379268 on novelty-induced locomotion, one between- and one within-subject-study were conducted each.

\section{Results}

\section{Sustained novelty-induced hyperlocomotion in CD2-KO} mice

Novelty-induced hyperlocomotion was assessed over a $2 \mathrm{~h}$ exploration time. A repeated-measures ANOVA over this period split into 245 -minute intervals revealed a significant effect of genotype $\left(P_{\text {genotype }}<0.0005\right)$ indicating that $\mathrm{KO}$ mice were hyperlocomotive (Fig. 1a, b; see Supplementary Table S1 for statistical details on this and subsequent results including $\mathrm{n}-$, F- and $\mathrm{t}$-values). Additionally, the number of rotations, an indicator of stereotypies, was significantly elevated in knockouts of both genders $\left(P_{\text {genotype }}=0.001\right.$; ANOVA, Fig. $\left.1 \mathrm{c}\right)$.

\section{Normal amphetamine-induced hyperlocomotion in CD2- KO mice}

In a similar locomotor activity paradigm, injection of $2 \mathrm{mg} / \mathrm{kg}$ amphetamine after an acclimatisation period of $30 \mathrm{~min}$ resulted in a significant subsequent increase of locomotion relative to the vehicle and $1 \mathrm{mg} / \mathrm{kg}$ amphetamine conditions in both genotypes $\left(P_{\text {dose }}<0.0005, P_{\text {ge- }}\right.$ notype $=0.015, \quad P$ genotype"dose $=0.957$, ANOVA; betweensubject design; Fig. 1d, e). Univariate ANOVA within each dose revealed that the higher locomotion of CD2knockouts relative to controls seen qualitatively at each dose, only reached significance after vehicle $(P=0.007)$ but not after amphetamine injections $(P>0.1)$. Likewise, when expressing locomotor activity post-amphetamine relative to locomotor activity before injection there was no difference between genotypes $(P=0.759)$, while the effect of dose remained $(P<0.0005$, ANOVA). This suggests that $\mathrm{CD} 2-\mathrm{KO}$ mice are similarly sensitive to amphetamine as controls; if anything, amphetamine aligns the wildtype with the knockout levels of locomotor activity (Fig. 1f).

\section{Incomplete reduction of novelty-induced locomotion by the $D 2 / 3 \mathrm{R}$ antagonist raclopride}

Signalling through dopamine-receptors of the D2-type (D2R) in the nucleus accumbens mediates the level of spontaneous locomotor drive in rodents ${ }^{29}$, and antagonism of these receptors is the suggested mechanism of the antipsychotic action of neuroleptics. We therefore tested, if the novelty-induced hyperlocomotion in CD2$\mathrm{KO}$ mice is indeed responsive to the $\mathrm{D} 2 \mathrm{R}$-antagonist raclopride. We first confirmed in a dose-response study in a separate group of male $\mathrm{C} 57 \mathrm{BL} / 6 \mathrm{~J}$ mice, that the doses $0.33,1$ and $3 \mathrm{mg} / \mathrm{kg}$ raclopride were effective in reducing hyperlocomotion induced either by novelty or by the NMDA-receptor blocker MK-801 (Fig. 1g). Using the two lowest effective doses 0.33 and $1 \mathrm{mg} / \mathrm{kg}$, we firstly performed a between-subject study in CD2-KO mice and controls. While both doses of raclopride significantly reduced locomotion (distance moved) within both genotypes, surprisingly, knockout mice remained significantly hyperlocomotive compared to wildtype controls injected with the same doses $(P<0.0005$, ANOVA; $P<0.05$ for ANOVAs at each dose, Fig. 1h). That means, although 0.33 and $1 \mathrm{mg} / \mathrm{kg}$ could reduce locomotion in knockouts to the level of vehicle-injected wildtypes, these doses did not alleviate the effect of genotype within each dose. Also, $0.33 \mathrm{mg} / \mathrm{kg}$ raclopride could significantly reduce the number of rotations in wildtype mice compared to vehicle $(P=0.004$, Tukey post-hoc test), but not in knockout mice $(P=0.646)$, suggesting that the drug is even less effective in the latter (Supplementary Table S1).

To investigate this further, a subset of mice were continued in a within-subject regime with injections of vehicle and the doses 0.11, 0.33, 1 and $3 \mathrm{mg} / \mathrm{kg}$. Again, at all doses, knockout mice remained hyperlocomotive compared to wildtype controls $(P<0.0005$, repeatedmeasures ANOVA; $P<0.05$ for univariate ANOVAs). Across all 5 doses a significant dose*genotype interaction was apparent $(P=0.033$, Fig. 1i), indicating a differential efficacy of raclopride. Expressing the amount of locomotion as percentage of the locomotion each mouse displayed in its vehicle-trial revealed that the higher doses, 1 and $3 \mathrm{mg} / \mathrm{kg}$, reduced novelty-induced locomotion significantly less in knockout mice compared to wildtype controls $(P<0.05$, ANOVA, Fig. $1 \mathrm{j})$, supporting the view that the drug is less effective in CD2 knockouts. This suggests that, while D2Rs may mediate baseline locomotion, there is an additional D2R-independent mechanism of increasing locomotor drive in CD2-knockouts. This is reminiscent of the hyperlocomotion produced by electrical $(20 \mathrm{~Hz})$ stimulation of the ventral hippocampus in rats, which is mediated by D1- rather than D2receptors ${ }^{5}$. 


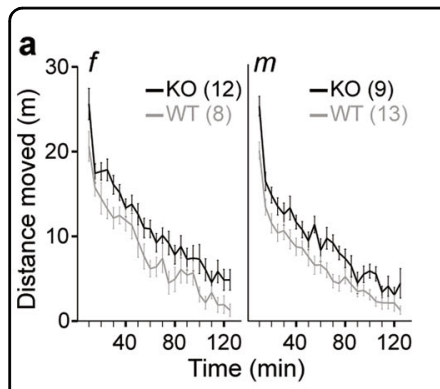

g

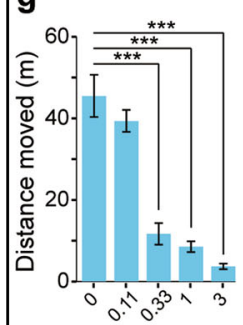

Raclopride (mg/kg)
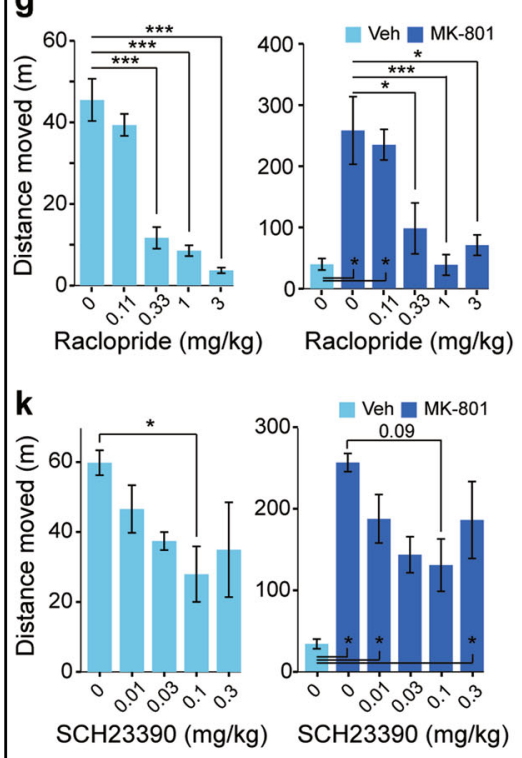

0
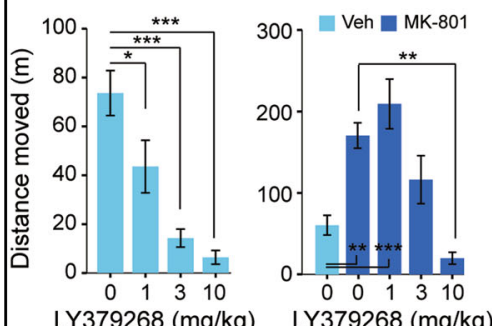
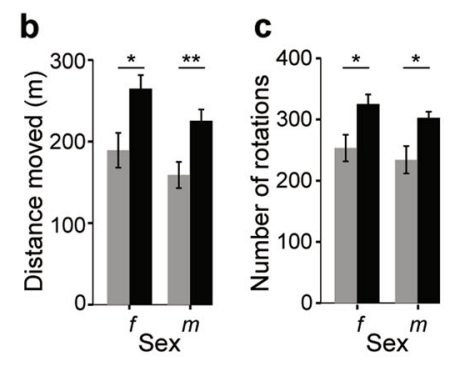

d Amphetamine dose $(\mathrm{mg} / \mathrm{kg})$

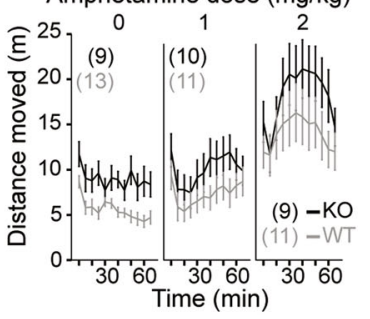

h

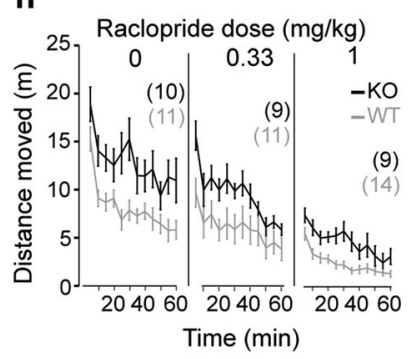

I

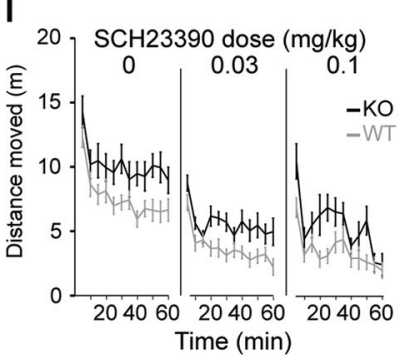

p

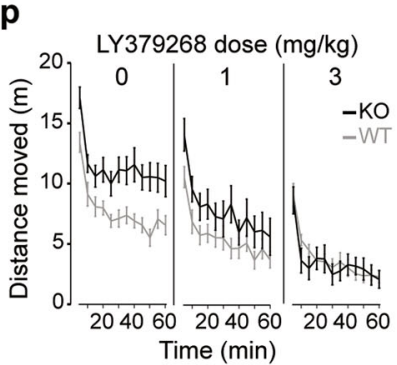

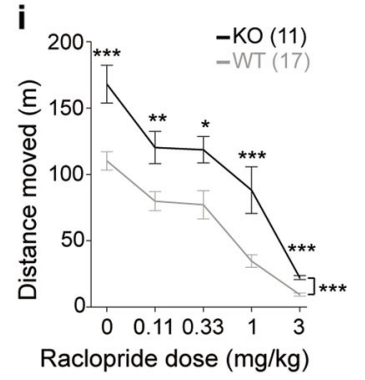

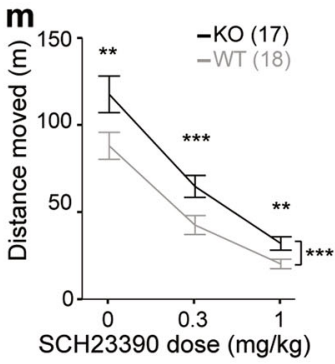

q

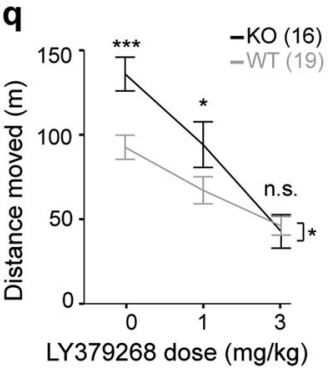

n
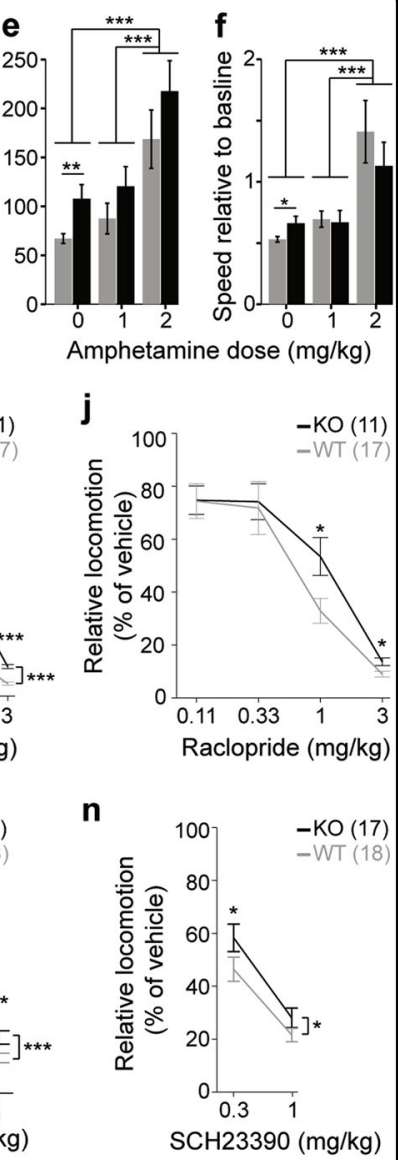

j
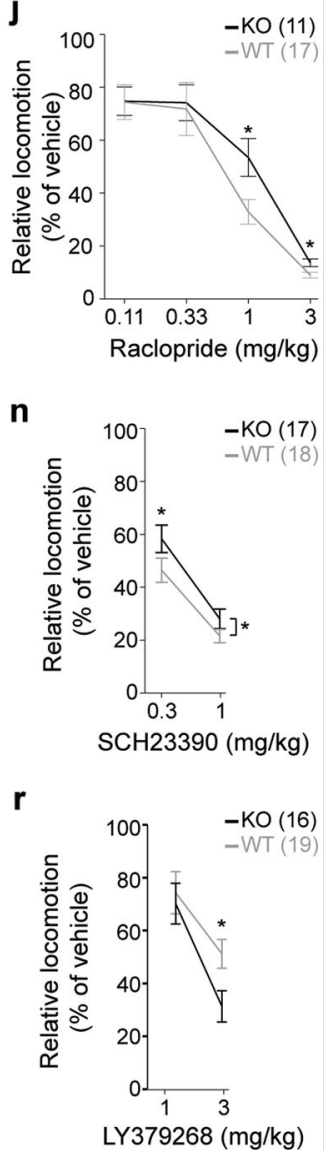

Fig. 1 Pharmacology of novelty-induced hyperlocomotion in CD2-KO mice. (a, b) Locomotion in a novel environment in 5 min intervals (a) and total over $2 \mathrm{~h}$ (b). (c) Number of rotations in the same experiment. (d, e) Locomotion after injection of vehicle (0), 1 or $2 \mathrm{mg} / \mathrm{kg}$ amphetamine in $5 \mathrm{~min}$ intervals (d) and in total over $2 \mathrm{~h}(\mathbf{e})$. (f) Same data as in (e) but expressed as speed $(\mathrm{m} / \mathrm{min}$ ) relative to the speed in the 30 min preceding the

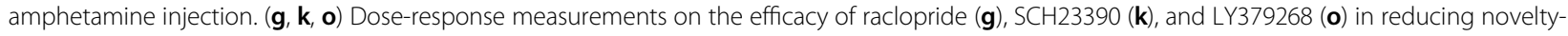
induced (left) and MK-801-induced (right) hyperlocomotion using the stated doses (x-axes); dark-blue indicates injections of $0.2 \mathrm{mg} / \mathrm{kg}$ MK-801, lightblue is vehicle. (h) Between-subject assessment of the efficacy of raclopride at the stated doses on novelty-induced locomotor activity in CD2-mice in 5 min intervals (total distance moved and statistics not shown). (i, $\mathbf{m}, \mathbf{q})$ Within-subject assessment of raclopride (i), SCH23390 (m), and LY379268 (q) at the stated doses on novelty-induced locomotion, plotting total distance moved in $1 \mathbf{h}$. (j, $\mathbf{n}, \mathbf{r})$ Within-subject assessment of raclopride (j), SCH23390 (n), and LY379268 (r) at the stated doses on novelty-induced locomotion, plotting distance moved in $1 \mathrm{~h}$ (as \%) relative to the vehicle (0) value in each animal. (I, p) Same data as in ( $\mathrm{m}$, q), within-subject assessment of SCH23390 (I) and LY379268 (p) at the stated doses on noveltyinduced locomotion, plotting distance moved in 5 min intervals. Wildtype controls, grey; CD2-KO, black; $f$, females, $m$, males; numbers in brackets denote $n$ for each group. All doses stated in $\mathrm{mg} / \mathrm{kg}$. ${ }^{*} P<0.05,{ }^{* *} P<0.01,{ }^{* * *} P \leq 0.001$. For trends, $P$ values are stated as numbers. Center values represent mean, Error bars, s.e.m. 


\section{Incomplete reduction of novelty-induced locomotion by the D1R antagonist SCH23390}

Therefore, we repeated this experiment with the dopamine D1-receptor (D1R) antagonist SCH23390. Assessing the efficacy of $0.011,0.033,0.1$ and $0.3 \mathrm{mg} / \mathrm{kg}$ against novelty- and MK-801-induced locomotion in a separate cohort of C57BL6/J males revealed that the two intermediate doses 0.033 and $0.1 \mathrm{mg} / \mathrm{kg}$ had the strongest, yet statistically moderate, effects (between-subject design; Fig. $1 \mathrm{k})$. Those two doses were tested against novelty-induced locomotion in CD2-KO mice and controls (within-subject design). While the drug significantly reduced locomotion dose-dependently in both genotypes, $\mathrm{KO}$ mice again remained hyperlocomotive relative to wildtype controls receiving the same dose $\left(P_{\text {genotype }}<0.0005, P_{\text {dose }}<0.0005\right.$, $P_{\text {dose"genotype }}=0.016$, repeated-measures ANOVA; Fig. 1l-m). Expressing locomotor activity under each dose relative to distance moved after injection of vehicle confirmed that the drug was less effective in reducing hyperlocomotion in CD2-KO mice compared to wildtypes $\left(P_{\text {genotype }}=0.02\right.$, repeated-measures ANOVA; Fig. $\left.1 \mathrm{n}\right)$.

Similar to the results obtained with D2R- and D1Rspecific antagonists, the antipsychotic clozapine, which acts as antagonist on all dopamine receptors, could reduce novelty-induced hyperlocomotion only at a relatively sedative dose $(3 \mathrm{mg} / \mathrm{kg})$ but not at an intermediate dose $(1 \mathrm{mg} / \mathrm{kg})$ in CD2-KO mice (see Supplementary Figure 1).

These data suggest that a largely dopamine-independent component of locomotor drive is elevated in CD2knockouts.

\section{Normalization of novelty-induced locomotion by the mGluR2/3 agonist LY379268}

Agonists of the metabotropic glutamate-receptor type 2/ 3 (mGluR2/3) have been proposed to be able to scale down glutamatergic transmission ${ }^{30}$, and one compound from this family, LY379268, has been shown to normalize ventral hippocampal hyperactivity evoked by chronic ketamine application in mice $^{2}$. In a separate cohort of adult male C57BL/6J mice, we found that a dose of $1 \mathrm{mg} / \mathrm{kg}$ LY379268 could reduce novelty-induced but not MK-801induced hyperlocomotion, while $3 \mathrm{mg} / \mathrm{kg}$ strongly reduced novelty-induced locomotion and moderately normalized MK-801-induced locomotion (Fig. 10). Testing both doses in CD2-KO mice (within-subject design), we found that $3 \mathrm{mg} / \mathrm{kg}$ can completely normalize noveltyinduced locomotion in $\mathrm{CD} 2-\mathrm{KO}$ mice relative to wildtype controls receiving the same dose $\left(P_{\text {genotype }}=0.941\right)$, while $1 \mathrm{mg} / \mathrm{kg}$ achieved a partial reduction $\left(P_{\text {genotype }}=\right.$ 0.029 at $1 \mathrm{mg} / \mathrm{kg}$ and $P_{\text {genotype }}<0.0005$ at vehicle, ANOVA, Fig. 1p, q). Indeed, LY379268 appeared to reduce locomotion even stronger in knockouts than in wildtypes $\left(P_{\text {genotype }}\right.$ dose $\left.=0.01\right)$ leading to significantly less relative locomotion at $3 \mathrm{mg} / \mathrm{kg}$ compared to wildtype controls
$\left(P_{\text {genotype }}=0.043\right.$, ANOVA; Fig. $\left.1 \mathrm{r}\right)$. We confirmed the finding of a significant interaction between dose (vehicle vs $3 \mathrm{mg} / \mathrm{kg}$ ) and genotype in a between-subject study in an extended cohort $\left(P_{\text {genotype"dose }}=0.015\right.$, n: $15 \mathrm{KO}, 20 \mathrm{WT}$; data not shown).

\section{CD2-KO mice are impaired in various forms of cognitive flexibility}

Impairments of executive function are a hallmark of schizophrenia, and reduced capacity to learn a rule-shift in a spatial associative learning task in the plus-maze has been demonstrated in $C c n d 2^{-/}$mice previously ${ }^{19}$. Firstly, we sought to confirm this finding. KO mice acquired a spatial long-term memory according to an egocentric rule similarly well as wildtypes: the number of trials needed to reach the criterion of $85 \%$ correct of the last consecutive 20 trials did not differ significantly between genotypes ( $P_{\text {genotype }}=0.232, t$-test $)$, and the learning curves were similar $\left(P_{\text {genotype }}=0.367\right.$, repeatedmeasures ANOVA over the second half of the number of training sessions needed on average to reach criterion; Fig. 2a-c; see Supplementary Table 3 for statistical analysis of this data and all subsequent datasets related to cognitive flexibility). This indicates, that $\mathrm{CD} 2-\mathrm{KO}$ mice can process spatial information and acquire a spatial memory largely normally. Once animals had reached criterion, the associative rule was shifted to an allo-centric one ${ }^{19}$. In this task, $\mathrm{KO}$ mice needed significantly more trials to reach criterion again $\left(P_{\text {genotype }}=0.04, t\right.$-test $)$ and average performance was lower compared to wildtypes $\left(P_{\text {genotype }}=\right.$ 0.048 , repeated-measures ANOVA over the second half of the training sessions needed on average to reach criterion; Fig. $2 \mathrm{~d}-\mathrm{f}$ ).

Once mice had reached criterion again, rule-reversal learning was assessed: the allo-centric spatial location that was associated with a reward was switched. Similarly to the rule-shift, $\mathrm{KO}$ mice required more trials than controls to reach criterion again $(P=0.021, t$-test $)$ and their performance was inferior $(P=0.01$, repeatedmeasures ANOVA over the second half of the number of training sessions needed on average to reach criterion). Note that no significant difference was seen when analysing all training sessions $(P=0.497$; Supplementary Table 3a).

However, rule-shift learning is usually attributed to prefrontal rather than hippocampal dysfunction ${ }^{31}$. Therefore, we sought confirmation of our surprising finding by conducting a newly developed operant-box paradigm which implements a rule-shift within the visual domain and thereby resembles the Wisconsin cardsorting test (which can be indicative of prefrontal dysfunction in humans; Fig. 2j): mice that had been trained up in the 5-choice-serial-reaction-time task (see below) in which a rewarded nose-poke is indicated by a cue light, 


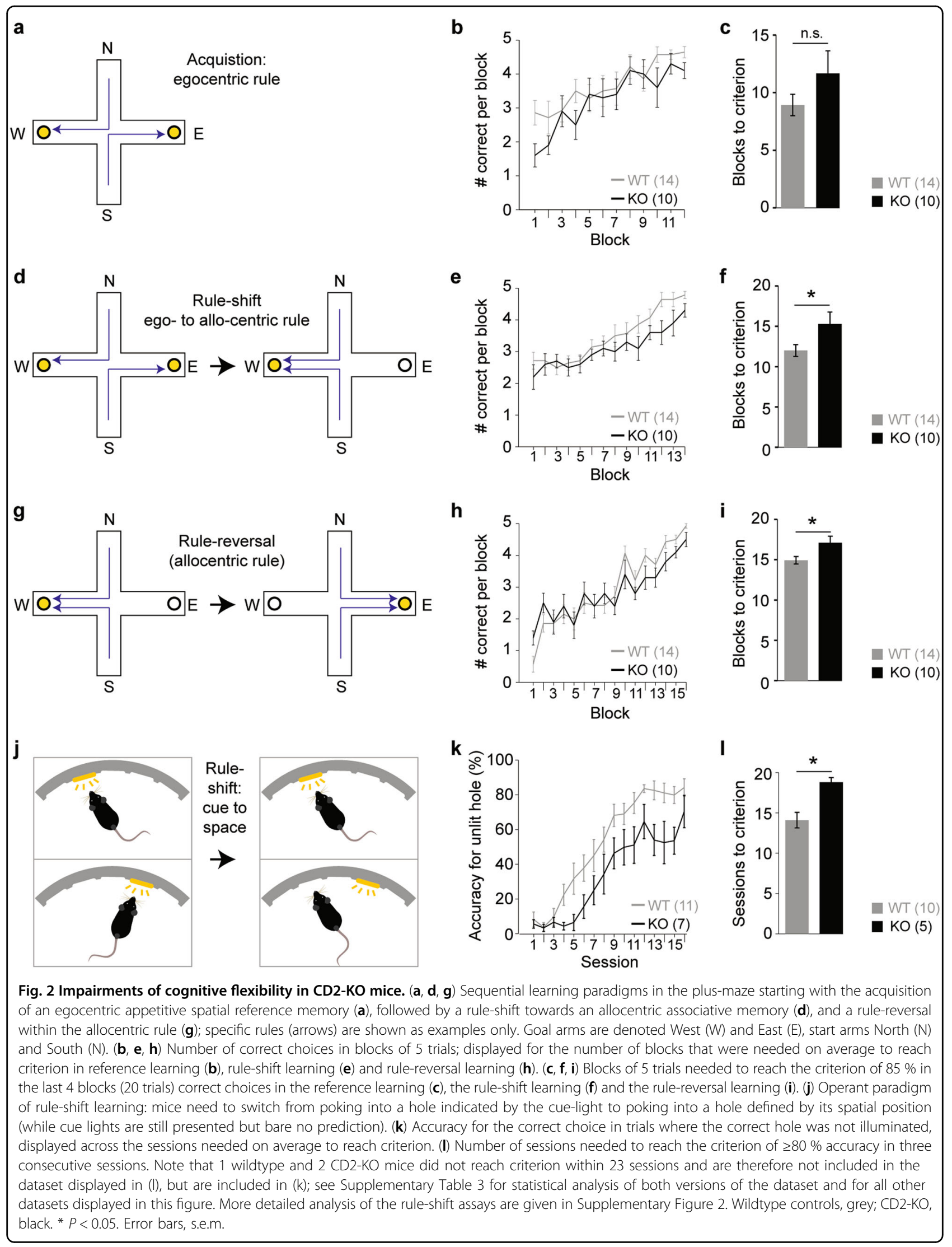


were subjected to a rule-shift whereby cue-lights were rendered meaningless while the fixed spatial location of the hole now indicated the correct choice. KO mice required significantly more trials to reach the criterion of $80 \%$ accuracy in the choice of the unlit rewarded hole on three consecutive days $(P=0.001, t$-test $)$ and showed lower accuracy during training $(P=0.009$, repeatedmeasures ANOVA over the second half of the training sessions needed on average to reach criterion, Fig. $2 k, 1)$.

Surprisingly, in both rule-shift assays, CD2-knockouts did not display a significantly higher number of perseverative errors. In contrast, their lower performance in the plus-maze was mainly due to erroneous choices that were never reinforced by the prior training, in line with a previous study ${ }^{19}$, while in the operant paradigm the difference was mainly due to a lower number of correct responses selectively only in those trials where the old and the new rule were in conflict (Supplementary Figure 2, Supplementary Table 3).

\section{CD2-KO mice are unimpaired in object-related and spatial short-term habituation}

Dysfunction of sustained and stimulus-specific attention (attentional control) have been related to schizophrenia $^{32,33}$, and could explain deficits in other cognitive tests conducted in this study. We first assessed a form of spatial short-term memory that is largely mediated by short-term habituation of attention using the spatial novelty-preference test in the $\mathrm{Y}$-maze ${ }^{34}$. In this test, habituation is measured as a preference for a novel as opposed to a familiar choice arm (or 'space'). In the sample phase, KO mice showed significant hyperlocomotion $(P=$ 0.018 , univariate ANOVA) but spent a similar amount of time in the to-be-familiar arm $(P=0.425)$ as wildtype mice, suggesting equal levels of exploration (Fig. 3a-c). In the test phase, mice of both genotypes explored the novel arm significantly more than the familiar arm, in terms of both entries and time $(P<0.0005$, repeated-measures ANOVA, Fig. 3d, e), and there were no genotype-related differences in the preference ratios for the novel arm $(P>$ 0.2, ANOVA; Fig. 3f). Additionally, preference ratios were significantly above chance level in both genotypes $(P<$ 0.005 , one-sample $t$-test).

Novel-object recognition represents an object-related version of the novelty-preference test of habituationdriven short-term memory (Fig. 3g) ${ }^{33,35}$. CD2-KO mice, compared to controls, showed strongly increased exploration of the objects in the sample phase and the test phase $(P<0.01$; ANOVA; Figure $3 \mathrm{~h}-\mathrm{j})$. However, mice of both genotypes showed significantly higher exploration of the novel compared to the familiar object in the test phase $(P<0.0005$, repeated-measures ANOVA), and did not differ in their preference for the novel object $(P>0.4$, ANOVA; Fig. 3k). In summary, this indicates, that $\mathrm{CD} 2-\mathrm{KO}$ mice are not impaired in short-term habituation of stimulus-specific attention.

\section{CD2-KO mice are not impaired in sustained attention, motivation, perseveration or impulse control}

To assess sustained attention, CD2-knockouts and wildtype controls were trained on the 5-choice serial reaction time task (5-CSRTT) through 4 stages of increasing difficulty, before being challenged with different protocols (see Supplementary Methods). Mice of both genotypes learned its basic operant cycle in the habituation training similarly well, in terms of required trials and obtained rewards (Supplementary Table 1a). In the baseline protocol of the 5-CSRTT, which was conducted with a stimulus duration (SD) of $4 \mathrm{~s}$ and an inter-trialinterval (ITI) of $5 \mathrm{~s}$, knockout mice appeared significantly better in the primary measure of attention, \% accuracy $(P$ $=0.038$; ANOVA), although in each of the gender groups alone there was no significant effect of genotype $(P>0.05$, $t$-test; Fig. $3 \mathrm{~m})$. In a measure of inattentiveness, \%omissions, female knockouts performed worse than female wildtype mice ( $P=0.005, t$-test), although, qualitatively, the opposite was observable in the male cohort, yielding a significant genotype"gender interaction $(P=0.006$, ANOVA; Fig. $3 \mathrm{n})$. The basic measure of motivation, reward latency, did not differ between genotypes $(P>0.3$, ANOVA), but females, irrespective of genotype, had a longer reward latency than males $\left(P_{\text {gender }}<0.05, P_{\text {gender* }}\right.$ genotype $=0.307$, ANOVA; Fig. 3o). Additionally, perseveration, indicated by unrewarded repetition of nosepokes into previously rewarded holes, was not significantly higher in CD2-KO mice compared to controls $(P=0.303$, ANOVA; Fig. 3p).

After baseline training, four challenge protocols were conducted, out of which three were targeted at sustained attention (shortening of the SD, distraction during the ITI, or both), and one at impulse control (increasing the ITI to $9 \mathrm{~s}$ ). Within 60 sessions, 2 wildtype males and 7 of the 13 involved females (KO and WT) did not reach the criterion in the baseline stage to proceed to challenge protocols, which were therefore only conducted in males. Each challenge protocol was conducted over two consecutive days and was preceded and succeeded by sessions with the baseline protocol. For analysis of each parameter, the values from the two challenge days were averaged and a repeated-measures ANOVA was calculated by including the performance of the preceding baseline session.

Regarding impulse control, all challenges increased the primary measure of motor impulsivity, \% premature responses $(P<0.0005$ for all challenges except the exclusive distraction $(P=0.015)$, repeated-measures ANOVA). Similarly, all challenges decreased attentional accuracy $(P<0.0005$ for all attention challenges, $P=0.001$ for the impulsivity challenge). A trend of slightly better attention 


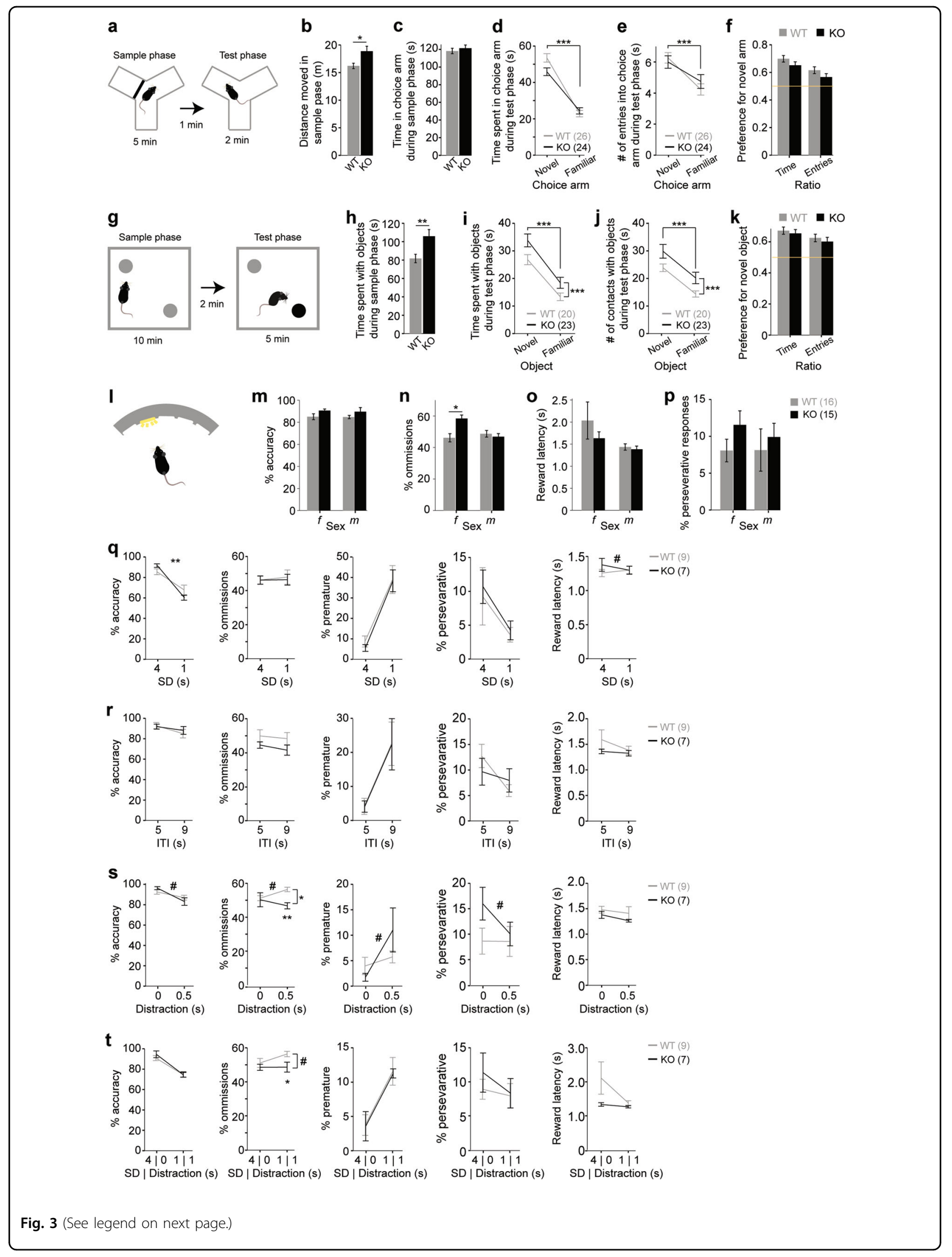


Fig. 3 Short-term habituation and sustained attention in CD2-KO mice. (a) Paradigm of the spatial-novelty preference test. (b) The total distance moved and (c) time spent in the to-be-familiar choice arm in the sample phase. (d) Time spent in the indicated choice arms and (e) number (\#) of entries into them during the test phase. (f) Preference for the novel arm according to either time or entry measures. (g) Paradigm of the novel-object recognition task; different colours indicate different types of objects. (h) Amount of time the animals investigated both objects in the sample phase. $(\mathbf{i}, \mathbf{j})$ Interaction with the novel and the familiar object in the test phase in terms of time (i) and number (\#) of contacts (j). (k) Preference for the novel object according to either time or entry measures. (I) Illustration of the 5-choice-serial-reaction-time task. (m-p) Performance in measures of attention (accuracy $(\mathbf{m})$, percent omissions $(\mathbf{n})$ ), incentive motivation (latency to reward receptacle, $(\mathbf{o})$ ), and perseveration (percent perseverative responses into the correct hole, $(\mathbf{p})$ ) in the baseline protocol ( $4 \mathrm{~s}$ stimulus duration, SD; $5 \mathrm{~s}$ inter-trial-interval, ITl; average of the last 4 sessions before the challenges started). (q-t) Measures of attention (accuracy, left; \%ommissions, second from left), impulsivity (percent premature responses relative to all initiated trials, middle), perseveration (percent perseverative responses, second from right) and motivation (reward latency, right) in various challenge protocols (right value in all subpanels, average of 2 consecutive sessions) relative to the baseline protocol immediately before the challenge (left value in all subpanels). Attention was challenged by a reduction of the SD to $1 \mathrm{~s}(\mathbf{q})$, a random distraction by switching of the house light for $0.5 \mathrm{~s}$ during the ITI $(\mathbf{s})$, and a combination of SD-reduction to $1 \mathrm{~s}$ and random switching the house light off for $1 \mathrm{~s}(\mathbf{t})$. Impulsivity was challenged by increasing the waiting time (ITI) until the holes were illuminated to $9 \mathrm{~s}(\mathbf{r})$. Wildtype controls, grey; CD2-KO, black; $f$, females, $m$, males; numbers in brackets denote $n$ for each group. ${ }^{*} P<0.1,{ }^{*} P<0.05,{ }^{* *} P<0.01,{ }^{* * *} P \leq 0.001$. In within-subject data (line graphs), symbols on horizontal lines indicate an effect of the within-subject factor (not drawn in (q-t) for clarity), symbols on vertical lines indicate effects of genotype across conditions (ANOVA), symbols on the connecting lines indicate an interaction between both factors, and symbols below the data point of the challenge conditions $(q-t)$ indicate a significant effect of genotype in the challenge data alone (t-test). The yellow lines indicate chance-level performance. Error bars, s.e.m.

in $\mathrm{CD} 2-\mathrm{KO}$ mice at baseline (see above) was seen in the baseline sessions of most protocols (Fig. 3q-t). However, in knockout mice attentional accuracy was affected significantly stronger by the first (and therefore unexpected) attentional challenge $\left(P_{\text {challenge"genotype }}=0.006\right)$, and a similar trend $\left(P_{\text {challenge"genotype }}=0.066\right)$ was seen with the first distraction challenge (Fig. 3q, s). However, that effect was absent at the later combined SD-reduction/distraction challenge (Fig. 3t), suggesting it does not reflect a genuine, reproducible attentional deficit. In line with this interpretation, CD2-KO mice even displayed significantly lower omission rates than controls under both distractionchallenge conditions $(P<0.05, t$-tests, Fig. $3 \mathrm{~s}, \mathrm{t})$.

Finally, there were no significant effects of genotype nor genotype ${ }^{*}$ challenge interactions $(P>0.05)$ detectable on any of the protocols in the measures reward latency, \% premature, or \%perseverative responses (Fig. 3q-t).

In summary, CD2-knockout mice appeared largely normal on all psychiatrically relevant parameters measured by the 5-CSRTT and were unimpaired in acquiring an associative memory during operant learning.

\section{CD2-KO mice show a working memory impairment that is resistant against LY379268}

To assess spatial working memory, mice were trained on a rewarded alternation (delayed-non-match-to-place) task on the T-maze over 5 days in a blocked paradigm with a delay (intra-trial interval) of $5 \mathrm{~s}$ and an inter-trial interval of $>3 \mathrm{~min}$. While female knockouts did not differ from controls in this initial training paradigm, male knockouts performed worse across those training sessions (females: $P_{\text {genotype }}=0.497$; males: $P_{\text {genotype }}=0.005$; both genders: $P_{\text {genotype }}=0.013, P_{\text {gender }}=0.002, P_{\text {genotype"gender }}$ $=0.123, \quad P_{\text {day }}=0.001$, repeated-measures ANOVA; Fig. $4 a-c)$. Subsequently, mice were challenged by different alterations of the paradigm, each performed in two consecutive sessions: firstly, the delay was maximally reduced (" $1 \mathrm{~s}$ ") and the paradigm changed to massed trials with an inter-trial interval of $25 \mathrm{~s}$, which had revealed subtle deficits in mice with PV-interneuron-specific NMDA receptor hypofunction previously ${ }^{27,36}$. Then, blocked paradigms with increasing delay $(5 \mathrm{~s}, 20 \mathrm{~s}, 60 \mathrm{~s})$ were conducted. Across paradigms and genders, knockout mice were impaired $(P<0.0005)$ and females of both genotypes performed significantly better than males $(P<$ 0.0005 ; repeated-measures ANOVA). When assessing individual challenges by univariate ANOVAs across genders, all protocols except the 60s-delay revealed significantly reduced performance in knockouts $(P<0.05$; Fig. 4d). However, when analysing females alone in the 60 s-protocol, again CD2-KOs performed significantly worse $(P=0.024, t$-test $)$, suggesting that the strongly reduced performance in male wildtype mice prevented the detectability of an effect of genotype across genders in this protocol (Fig. 4e).

We next examined, if the mGlu2/3R-agonist LY379268 was also effective against the working memory deficit in $\mathrm{CD} 2-\mathrm{KO}$ mice as it was against their hyperlocomotion. However, across doses, the lower performance of knockouts remained apparent $\left(P_{\text {genotype }}=0.007, P>0.05\right.$ for effects of dose, gender and all interactions, repeatedmeasures ANOVA, within-subject design, Fig. 4f).

These data imply that CD2-KO mice show a delayindependent impairment of working memory, which is resistant against treatment with mGluR2/3-agonists.

\section{CD2-KO mice show normal unconditioned anxiety}

During the testing of novelty-induced locomotion in an open field (see above), we found no effect of genotype on the average distance to the cage walls $(P>0.1$ for all 

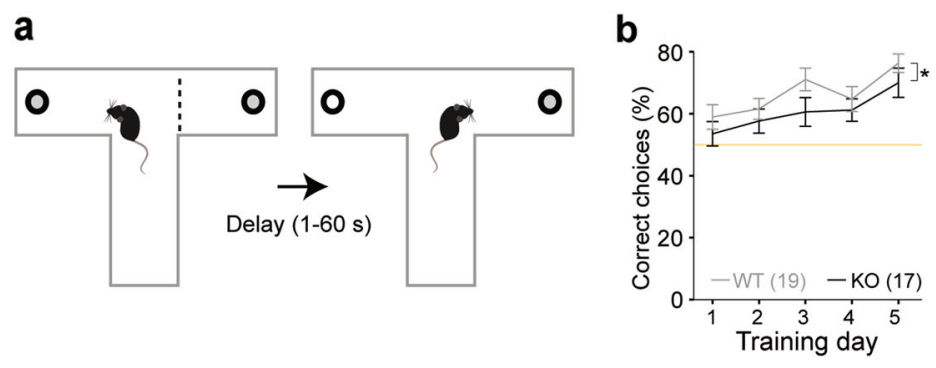
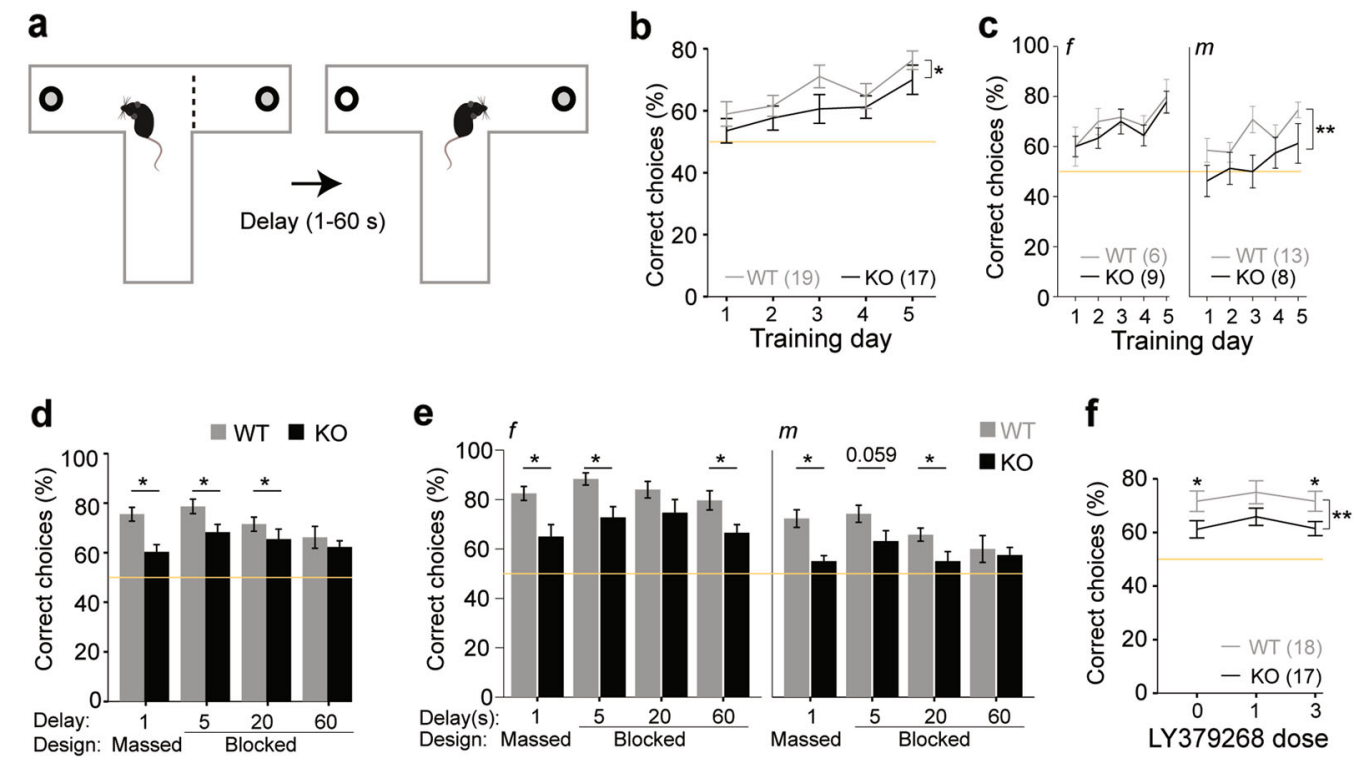

Fig. 4 Impaired spatial working memory in CD2-KO mice. (a) Paradigm of the spatial working memory test. (b, c) Performance (correct choices out of 10 trials, in \%) over the 5 initial training days shows a significant effect of genotype across the total cohort (b), which is driven by the male subgroup (c, right), rather than the females (c, left). (d, e) Performance under 4 different challenge protocols conducted in the same order as plotted with 2 consecutive days per protocol (averaged for the display) shown for the whole cohort (d) and the genders separately (e); protocols differed by their design (massed vs blocked) and the delay between sample and choice trial as indicated on the $x$-axes. (f) After a re-training (identical to the previous one (b), data not shown) either vehicle (0), 1 or 3 mg/kg LY379268 was injected into CD2-KO mice and controls prior to testing sessions in the massed design ( $1 \mathrm{~s}$ delay). Performance (correct choices out of 10 trials, in \%) is shown for both genders combined. Wildtype controls, grey; CD2$\mathrm{KO}$, black; $f$, females, $m$, males; numbers in brackets denote $n$ for each group. ${ }^{*} P<0.05,{ }^{*} P<0.01$. In within-subject data (line graphs), symbols above data points indicate an effect of group in the indicated condition, symbols on vertical lines indicate effects of genotype across conditions. The yellow lines indicate chance-level performance. Error bars, s.e.m.

factors, ANOVA, Fig. 5a), suggesting similar levels of unconditioned anxiety. Likewise, on the elevated plusmaze, animals of both genotypes spent significantly more time in the closed compared to the open arms $\left(P_{\text {arm }}<\right.$ 0.0005, repeated-measures ANOVA, Fig. 5b). Accordingly, the preference ratio for the open arm (time spent in open arms divided by time spent in all arms) was not significantly different between genotypes $(P=0.488)$, but was significantly lower in females compared to males $(P=0.003$, univariate ANOVA, Fig. 5c). Preference ratios calculated from the number of entries did not differ between any groups $(P>0.1$, ANOVA, Fig. $5 \mathrm{~d})$. This indicates that anxiety is unaltered in CD2-knockouts.

\section{CD2-KO mice show transient anhedonia in the sucrose preference test}

Analysing the preference ratio for sucrose (consumed volume of liquid from sucrose bottle relative to total consumed liquid) over five nights, we found a strong effect of night (indicating sucrose concentration; $P<$ $0.0005)$ but also a night"genotype interaction $(P=0.007)$ without an effect of genotype $(P=0.647$, repeatedmeasures ANOVA), suggesting that both genotypes develop a strong preference for sucrose but with a different trajectory (Fig. 5e-g). This divergence, resulted largely from the first encounter with sucrose (night 2), where an interaction between genotype and bottlecontent (water vs sucrose, $P=0.001$ ) on the consumed liquid volume, as well as a significantly reduced consumed volume of sucrose solution $(P=0.014)$ and sucrose preference in knockouts $(P=0.012$, ANOVAs) were apparent. Such a phenotype of mild anhedonia at the beginning of sucrose-preference testing in $\mathrm{CD} 2-\mathrm{KO}$ mice was described previously ${ }^{21}$.

\section{Nest building is normal in CD2-KO mice}

We measured nest building as a compound indicator of hippocampus-dependent social and motivational functioning ${ }^{28}$. Neither the nest score nor the amount of untorn material (bits of $>0.1 \mathrm{~g}$ ) were significantly different between genotypes $(P>0.2$ for within-gender comparisons of both parameters, Mann-Whitney-U-test, Fig. $5 \mathrm{~h}-\mathrm{j})$.

CD2-KO mice show enhanced reciprocal social interaction

To assess social interaction, test mice were left to interact with younger, same-sex C57BL/6 stimulus mice in novel cages. Surprisingly, the total interaction time and the number of interactions were significantly higher in CD2-KO mice compared to wildtypes $\left(P_{\text {genotype }}<0.05\right.$, 


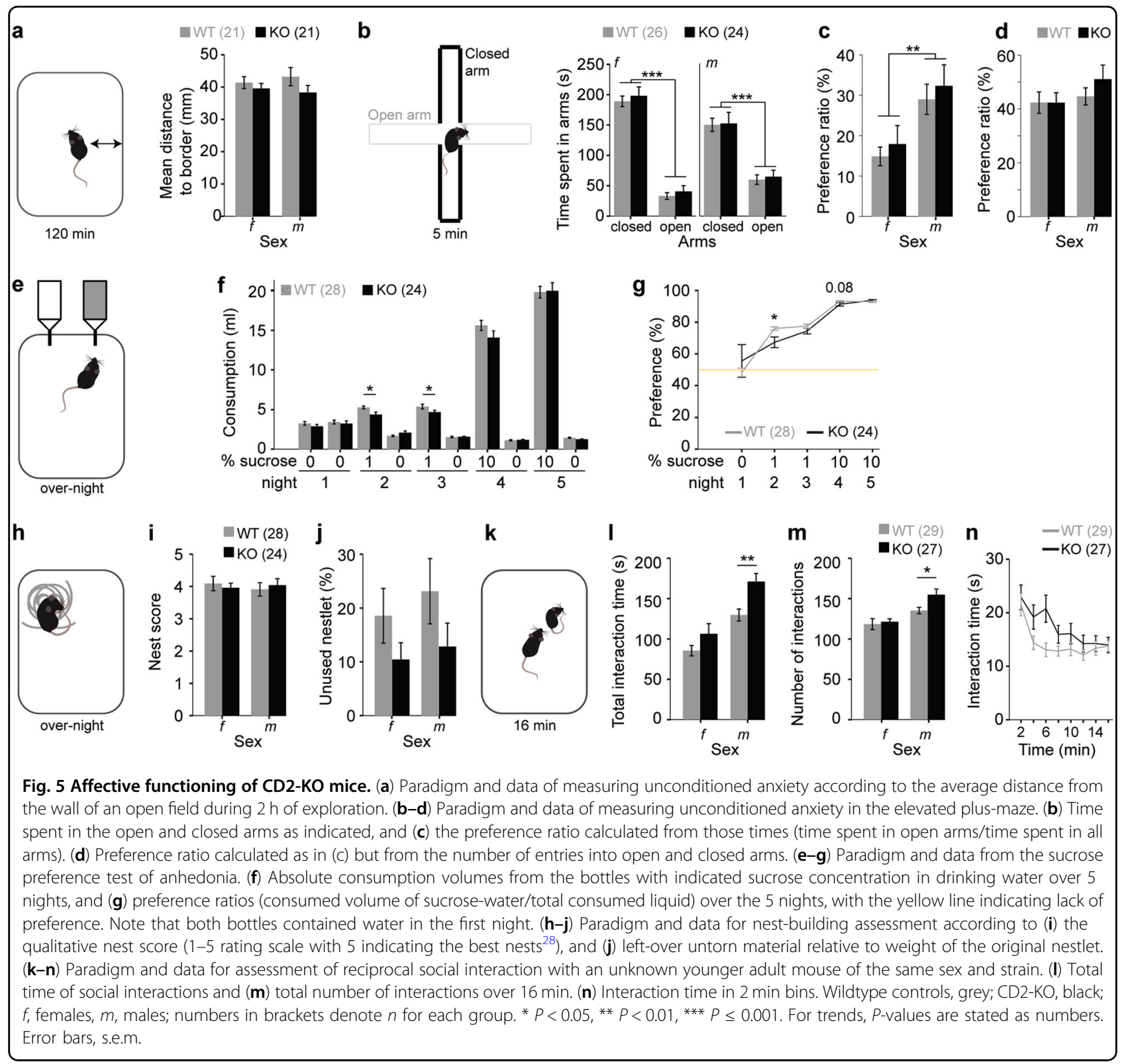

ANOVA; Fig. 5k, l). Males showed more interaction than females $\left(P_{\text {gender }}<0.0005\right.$ for both parameters), and the increased interaction in $\mathrm{CD} 2-\mathrm{KO}$ mice reached significance only in the male subgroup in both parameters $\left(P_{\text {males }}<0.02, P_{\text {females }}>0.1, t\right.$-tests $)$. This difference was mainly driven by the early stages of the test, in which CD2-KO mice showed a sustained high level of interaction time $\left(P_{\text {interval "genotype }}=0.003\right.$, repeated-measures ANOVA; Fig. $5 \mathrm{n})$.

\section{Discussion}

We here did a comprehensive behavioural assessment of deficits across the three symptom domains of schizophrenia in the Cyclin-D2-KO mouse model of ventral hippocampal hyperactivity. It was shown previously, that this mouse line gains its construct validity as a model of schizophrenia from (1) a hypofunction of parvalbuminpositive interneurons, (2) reduced hippocampal volume, and (3) a lack of adult neurogenesis - three cellular pathologies observed in schizophrenia ${ }^{2,15,16,20-22,37}$. It also displays a mesolimbic hyperdopaminergic state, behavioural hyperactivity, and elevated activity of hippocampal subfields CA1-3, all of which could be ameliorated by increasing inhibition in the ventral hippocampus in a previous study ${ }^{15}$. This prior work suggests that CD2-KOmice could provide a unique rodent model to understand the relationship between the endophenotype of hippocampal hyperactivity - analogously observed in patients 
with schizophrenia at baseline $\mathrm{e}^{2,3,38-41}$ and during sensory processing $^{42,43}$ - and symptoms of this disease. Hippocampal hyperactivity correlates with positive, but also with cognitive symptoms in patients, while conflicting results exist regarding the negative symptom domain ${ }^{3,41}$.

Regarding cognitive symptoms, our data demonstrates impairments of CD2-knockouts in cognitive flexibility, affecting both rule-shift and rule-reversal learning (extending earlier findings, see Table 1). It should be noted though, that none of the paradigms used here although in similar form widely applied in rodent research - involve a fast learning process as is common in cognitive flexibility in humans, raising the possibility that they rather assess changes of habits. Likewise, the anatomical basis of the observed deficits is unclear: while the hippocampus has been implied in rule-reversal learning directly $^{44,45}$ (just as the orbito-frontal cortex ${ }^{46}$ ), its effect on rule-shift learning might be mediated through its influence on the medial prefrontal cortex, as suggested by a similar observation in a rat model with a ventralhippocampal lesion ${ }^{47}$. Importantly though, perseverative errors, which are the dominant error among patients with frontal lobe damage - but not among patients with schizophrenia - on the Wisconsin card-sorting test of cognitive flexibility ${ }^{48}$, did not significantly contribute to the impairments seen in CD2-knockouts.

Our data also uncovers a previously unknown impairment in spatial working memory in CD2-knockouts. This aligns with a negative correlation between hippocampal hyperactivity and working memory seen in schizophrenia patients ${ }^{41}$, and with impairments of short-term memory produced by broad chemogenetic ${ }^{12}$ or pharmacological ${ }^{13}$ hippocampal disinhibition in rodents.

In contrast to patient data (and the just-cited rat study), however, we could not find an indication that CD2-KO mice are consistently impaired in sustained attention: they showed slightly improved sustained accuracy at baseline, which was then, however, somewhat stronger disrupted by some unexpected challenges. At the same time, knockouts were resistant against the increase in inattentiveness (omitted responses) evoked by distraction challenges in wildtype controls. Both findings are in line with the notion that schizophrenia might be associated with higher but inflexible ("sticky") attention, rather than blunt inattentiveness ${ }^{33}$.

Knock-out mice were also unimpaired in short-term habituation of stimulus-specific attention. This psychological mechanism has been suggested to underlie aberrant salience ${ }^{33}$, and thereby psychosis ${ }^{49}$, which implies that these mice probably do not model the psychotic state of schizophrenia. This aligns with their physiological profile of showing hyperactivity in CA1 but not in the subiculum $^{15}$ (see Introduction), and with their normal response to amphetamine (however, see ${ }^{15}$ ).
Nevertheless, CD2-KO mice did display robust noveltyinduced hyperlocomotion, which is usually considered a rodent correlate of the positive symptom domain (note however, that individual positive symptoms can appear in prodromal patients ${ }^{50}$, and that the extent of hyperlocomotion in this mouse line appears considerably smaller than in a mouse model with impaired short-term habituation, the Gria1-knockout mouse ${ }^{33,34,51}$ ). Interestingly, the relative elevation of locomotion compared to controls was maintained even after treatment with D1R- or D2Rantagonists, in line with the unresponsiveness of baseline hippocampal hyperactivity to antipsychotic medication in patients ${ }^{3}$. In contrast, novelty-induced hyperlocomotion was completely normalized by the mGluR2/3-agonist LY379268, supporting the notion, that hippocampal glutamatergic activity can be scaled down by this pharmacological intervention ${ }^{2}$. This argues for the further clinical exploration of the anti-psychotic efficacy of mGluR2/3enhancement ${ }^{52}$, especially in cases where positive symptoms remain resistant against D1R/D2R-related neuroleptics.

Furthermore, CD2-knockouts displayed only minor abnormalities in the negative symptom domain, including enhanced social interaction, and transient anhedonia. Motor impulsivity, anxiety, and perseveration were normal. Generally, their deficit profile resembles schizophrenia - especially its prodrome - more than any other psychiatric disorder. Based on the presented phenotyping, this mouse model appears well-suited to develop therapeutical interventions targeted at cognitive deficits caused by an overactive hippocampus, and, more generally, at halting the transition from the prodromal to the psychotic state $^{4}$.

\section{Acknowledgements}

We thank Bastiaan van der Veen and Kasyoka Kilonzo for logistical support, Chris Barkus for providing Med-PC code, and Alexa Hagedorn, Ekaterina Merkel and Olena Sakk for assistance with colony maintenance, the animal licence and related animal work.

\section{Funding \\ This work was funded by the Institute of Applied Physiology (UIm University), and by grants from the Juniorprofessorship programme of Baden- \\ Württemberg (D.K.) and the Else-Kröner-Fresenius/German-Scholars- \\ Organization Programme for excellent medical scientists from abroad (D.K.).}

\section{Author contributions}

C.M.G., S.A., St.S. and D.K. did experiments. J.T. programmed operant box paradigms. P.S. and B.L. contributed essential resources. D.K. designed the study and wrote the manuscript, which was revised by all authors.

\section{Author details}

${ }^{1}$ Institute for Applied Physiology, UIm University, Ulm, Germany. ${ }^{2}$ Dana-Farber Cancer Institute and Harvard Medical School, Boston, MA, USA

\section{Conflict of interest}

The authors declare that they have no conflict of interest. 


\section{Publisher's note}

Springer Nature remains neutral with regard to jurisdictional claims in published maps and institutional affiliations.

Supplementary Information accompanies this paper at (https://doi.org/ 10.1038/s41398-018-0268-6).

Received: 13 May 2018 Revised: 8 August 2018 Accepted: 10 September 2018

Published online: 09 October 2018

\section{References}

1. Bolkan, S. S., De Carvalho, F. D. \& Kellendonk, C. Using human brain imaging studies as a guide toward animal models of schizophrenia. Neuroscience. https:/doi.org/10.1016/j.neuroscience.2015.05.055 (2015).

2. Schobel, S. A. et al. Imaging patients with psychosis and a mouse model establishes a spreading pattern of hippocampal dysfunction and implicates glutamate as a driver. Neuron 78, 81-93 (2013).

3. Schobel, S. A. et al. Anterior hippocampal and orbitofrontal cortical structural brain abnormalities in association with cognitive deficits in schizophrenia. Schizophr. Res. 114, 110-118 (2009).

4. Moghaddam, B. A mechanistic approach to preventing schizophrenia in atrisk individuals. Neuron 78, 1-3 (2013).

5. Taepavarapruk, P., Floresco, S. B. \& Phillips, A. G. Hyperlocomotion and increased dopamine efflux in the rat nucleus accumbens evoked by electrical stimulation of the ventral subiculum: role of ionotropic glutamate and dopamine D1 receptors. Psychopharmacol. (Berl.) 151, 242-251 (2000).

6. Howland, J. G., MacKenzie, E. M., Yim, T. T., Taepavarapruk, P. \& Phillips, A. G. Electrical stimulation of the hippocampus disrupts prepulse inhibition in rats: frequency- and site-dependent effects. Behav. Brain. Res. 152, 187-197 (2004).

7. Bast, T., Zhang, W.-N., Heidbreder, C. \& Feldon, J. Hyperactivity and disruption of prepulse inhibition induced by $\mathrm{N}$-methyl-d-aspartate stimulation of the ventral hippocampus and the effects of pretreatment with haloperidol and clozapine. Neuroscience 103, 325-335 (2001).

8. Lodge, D. J. \& Grace, A. A. Aberrant hippocampal activity underlies the dopamine dysregulation in an animal model of schizophrenia. J. Neurosci. 27, 11424-11430 (2007).

9. Lodge, D. J. \& Grace, A. A. Gestational methylazoxymethanol acetate administration: A developmental disruption model of schizophrenia. Behav. Brain. Res. 204, 306-312 (2009).

10. Pouzet, B., Zhang, W. N., Weiner, I., Feldon, J. \& Yee, B. K. Latent inhibition is spared by $n$-methyl-d-aspartate (nmda)-induced ventral hippocampal lesions, but is attenuated following local activation of the ventral hippocampus by intracerebral nmda infusion. Neuroscience 124, 183-194 (2004).

11. Lisman, J. E. et al. Circuit-based framework for understanding neurotransmitter and risk gene interactions in schizophrenia. Trends Neurosci. 31, 234-242 (2008).

12. Nguyen, R. et al. Parvalbumin and GAD65 interneuron inhibition in the ventral hippocampus induces distinct behavioral deficits relevant to schizophrenia. J. Neurosci. 34, 14948-14960 (2014).

13. McGarrity, S., Mason, R., Fone, K. C., Pezze, M. \& Bast, T. Hippocampal neural disinhibition causes attentional and memory deficits. Cereb. Cortex N. Y N. 1991 27, 4447-4462 (2017).

14. Sicinski, P. et al. Cyclin D2 is an FSH-responsive gene involved in gonadal cell proliferation and oncogenesis. Nature 384, 470-474 (1996).

15. Gilani, A. I. et al. Interneuron precursor transplants in adult hippocampus reverse psychosis-relevant features in a mouse model of hippocampal disinhibition. Proc. Natl. Acad. Sci. 111, 7450-7455 (2014).

16. Glickstein, S. B. et al. Selective cortical interneuron and GABA deficits in cyclin D2-null mice. Development 134, 4083-4093 (2007).

17. Bast, T., Pezze, M. \& McGarrity, S. Cognitive deficits caused by prefrontal cortical and hippocampal neural disinhibition. Br. J. Pharmacol. 174, 3211-3225 (2017).

18. Garthe, A., Huang, Z., Kaczmarek, L., Filipkowski, R. K. \& Kempermann, G. Not all water mazes are created equal: cyclin D2 knockout mice with constitutively suppressed adult hippocampal neurogenesis do show specific spatial learning deficits. Genes. Brain. Behav. 13, 357-364 (2014).

19. Paskewitz, S., Weiss, S., Inbar, B. P., Ross, M. E. \& Moore, H. Alter. Spat. Learn. mice a deficit hippocampal parvalbumin-Express. GABA-ergic Interne. leads Apparent Cogn. inflexibility. 52, 20/U31 (2014).
20. Jaholkowski, P. et al. New hippocampal neurons are not obligatory for memory formation; cyclin D2 knockout mice with no adult brain neurogenesis show learning. Learn. Mem. 16, 439-451 (2009).

21. Jedynak, P. et al. Lack of cyclin D2 impairing adult brain neurogenesis alters hippocampal-dependent behavioral tasks without reducing learning ability. Behav. Brain. Res. 227, 159-166 (2012).

22. Reif, A. et al. Neural stem cell proliferation is decreased in schizophrenia, but not in depression. Mol. Psychiatry 11, 514-522 (2006).

23. Song, J. et al. Parvalbumin interneurons mediate neuronal circuitryneurogenesis coupling in the adult hippocampus. Nat. Neurosci. 16, 1728-1730 (2013)

24. Temprana, S. G. et al. Delayed coupling to feedback inhibition during a critical period for the integration of adult-born granule cells. Neuron 85, 116-130 (2015).

25. Toni, N. \& Schinder, A. F. Maturation and functional integration of new granule cells into the adult hippocampus. Cold Spring Harb. Perspect. Biol. 8, a018903 (2016).

26. Crawley, J. N. Behavioral phenotyping of transgenic and knockout mice: experimental design and evaluation of general health, sensory functions, motor abilities, and specific behavioral tests1Published on the World Wide Web on 2 December 1998.1. Brain Res. 835, 18-26 (1999).

27. Bygrave, A. M. et al. Knockout of NMDA-receptors from parvalbumin interneurons sensitizes to schizophrenia-related deficits induced by MK-801. Transl. Psychiatry 12, e778 (2016).

28. Deacon, R. M. Assessing nest building in mice. Nat. Protoc. 1, 1117-1119 (2006).

29. Hauber, W. \& Münkle, M. Motor depressant effects mediated by dopamine D2 and adenosine A2A receptors in the nucleus accumbens and the caudateputamen. Eur. J. Pharmacol. 323, 127-131 (1997).

30. Imre, G. The Preclinical Properties of a Novel Group II Metabotropic Glutamate Receptor Agonist LY379268. Cns. Drug. Rev. 13, 444-464 (2007).

31. Cho, K. K. A. et al. Gamma Rhythms Link Prefrontal Interneuron Dysfunction with Cognitive Inflexibility in Dlx5/6+/- Mice. Neuron 85, 1332-1343 (2015).

32. Millan, M. J. et al. Cognitive dysfunction in psychiatric disorders: characteristics, causes and the quest for improved therapy. Nat. Rev. Drug. Discov. 11, 141-168 (2012).

33. Barkus, C. et al. What causes aberrant salience in schizophrenia? A role for impaired short-term habituation and the GRIA1 (GluA1) AMPA receptor subunit. Mol. Psychiatry 19, 1060-1070 (2014).

34. Sanderson, D. J. \& Bannerman, D. M. The role of habituation in hippocampusdependent spatial working memory tasks: Evidence from GluA1 AMPA receptor subunit knockout mice. Hippocampus 22, 981-994 (2012).

35. Sanderson, D. J. et al. Deletion of the GluA1 AMPA receptor subunit impairs recency-dependent object recognition memory. Learn. Mem. 18, 181-190 (2011).

36. Carlen, M. et al. A critical role for NMDA receptors in parvalbumin interneurons for gamma rhythm induction and behavior. Mol. Psychiatry 17, 537-548 (2012).

37. Harrison, P. J. GABA circuitry, cells and molecular regulation in schizophrenia: Life in the graveyard. Schizophr. Res. 167, 108-110 (2015).

38. Talati, P. et al. Increased hippocampal CA1 cerebral blood volume in schizophrenia. Neuroimage Clin. 5, 359-364 (2014).

39. Talati, P., Rane, S., Skinner, J., Gore, J. \& Heckers, S. Increased hippocampal blood volume and normal blood flow in schizophrenia. Psychiatry Res. 232, 219-225 (2015).

40. Medoff, D. R., Holcomb, H. H., Lahti, A. C. \& Tamminga, C. A. Probing the human hippocampus using rCBF: contrasts in schizophrenia. Hippocampus 11, 543-550 (2001).

41. Tregellas, J. R. et al. Intrinsic hippocampal activity as a biomarker for cognition and symptoms in schizophrenia. Am. J. Psychiatry 171, 549-556 (2014).

42. Holt, D. J. et al. Sustained activation of the hippocampus in response to fearful faces in schizophrenia. Biol. Psychiatry 57, 1011-1019 (2005).

43. Tregellas, J. R., Ellis, J., Shatti, S., Du, Y. P. \& Rojas, D. C. Increased hippocampal, thalamic, and prefrontal hemodynamic response to an urban noise stimulus in schizophrenia. Am. J. Psychiatry 166, 354-360 (2009).

44. Taylor, A. M. et al. Hippocampal NMDA receptors are important for behavioural inhibition but not for encoding associative spatial memories. Philos. Trans. R. Soc. Lond. B. Biol. Sci. 369, 20130149 (2014).

45. Malá, H. et al. Prefrontal cortex and hippocampus in behavioural flexibility and posttraumatic functional recovery: reversal learning and set-shifting in rats. Brain Res. Bull. 116, 34-44 (2015). 
46. Alm K. H., Rolheiser T., Mohamed F. B., Olson I. R. Frontotemporal white matter connectivity predicts reversal learning errors. Front. Hum. Neurosci. 2015; 9. https://doi.org/10.3389/ fnhum.2015.00343.

47. Placek, K., Dippel, W. C., Jones, S. \& Brady, A. M. Impairments in set-shifting but not reversal learning in the neonatal ventral hippocampal lesion model of schizophrenia: further evidence for medial prefrontal deficits. Behav. Brain. Res. 256, 405-413 (2013).

48. Laws, K. R. A Meta-analytic Review of Wisconsin Card Sort Studies in Schizophrenia: General Intellectual Deficit in Disguise? Cogn. NeuroPsychiatry 4, 1-30 (1999).

49. Kapur, S. Psychosis as a state of aberrant salience: a framework linking biology, phenomenology, and pharmacology in schizophrenia. Am. J. Psychiatry 160, 13-23 (2003).
50. Tso, I. F. et al. Factor Analysis of the Scale of Prodromal Symptoms: Data from the Early Detection and Intervention for the Prevention of Psychosis Program (EDIPPP). Early Interv. Psychiatry 11, 14-22 (2017).

51. Sanderson, D. J., Sprengel, R., Seeburg, P. H. \& Bannerman, D. M. Deletion of the GluA1 AMPA receptor subunit alters the expression of short-term memory. Learn. Mem. 18, 128-131 (2011).

52. Patil, S. T. et al. Activation of $\mathrm{mGlu2/3}$ receptors as a new approach to treat schizophrenia: a randomized Phase 2 clinical trial. Nat. Med. 13, 1102-1107 (2007).

53. Urbach, A. et al. Cyclin D2 knockout mice with depleted adult neurogenesis learn Barnes maze task. Behav. Neurosci. 127, 1-8 (2013).

54. Jedynak, P., Kos, T., Sandi, C., Kaczmarek, L. \& Filipkowski, R. K. Mice with ablated adult brain neurogenesis are not impaired in antidepressant response to chronic fluoxetine. J. Psychiatr. Res. 56, 106-111 (2014). 